\section{Airborne Geiger-Mode LiDAR for Large-Scale, High-Resolution Wide-Area Mapping}

Gl_Forum 2016, Vol.1

Page: 85-93

Short Paper

Corresponding Author:

thomas.bahr@harris.com

DOI: 10.1553/giscience2016_01_s85

\author{
Thomas Bahr' and Philip Smith² \\ 1 Harris Corporation, Gilching, Germany \\ 2 Harris Corporation, Melbourne, Florida, U.S.A.
}

\begin{abstract}
This presentation describes a new solution for geospatial Geiger-mode Avalanche Photodiode (GmAPD) LiDAR - a suite of sensors, processing tools and analytics born from a 15-year GmAPD legacy within certain DoD environments that will bring a significant increase in production efficiency to the commercial market. The objective of the sensor design was to support USGS LiDAR Quality Level 1 (and higher) data production over large areas from pressurized, fixed-wing aircrafts flying at altitudes of between $4 \mathrm{~km}$ and $10 \mathrm{~km}$ AGL. Topics to be covered include: 1) why GmAPD sensing is a significant advance in LiDAR collection efficiency, 2) how the ITI-1000 GmAPD LiDAR sensor supports costeffective, wide-area, high-density collection, with coverage rates much greater than 1,000 $\mathrm{km}^{2} / \mathrm{hr}$, and 3) how a high-volume production system, including noise reduction and photogrammetric registration techniques, is designed to reduce the production time for the GmAPD 3D point clouds while increasing data accuracy.
\end{abstract}

\title{
Keywords:
}

Geiger-mode LiDAR sensor; avalanche photodiode detector; production system; photogrammetry; point clouds

\section{Introduction}

Since the first instruments were made available in the mid-1990s, the commercial airborne LiDAR market has seen impressive growth. As LiDAR point cloud data has become more widely available, its use to support diverse industries ranging from defence to environmental protection has continued to grow as well. However, for the airborne market to continue to expand and diversify into more demanding applications, it must overcome its biggest barrier - the costs associated with large-scale, high-density, accurate LiDAR data acquisition and production (CARY 2009).

As a solution to these demands, LiDAR systems built with Geiger-mode Avalanche Photodiode (GmAPD) detectors provide significant efficiency gains for airborne mapping when compared with currently available linear-mode sensors. Because GmAPD detectors are 
physically compact and single-photon sensitive, they can be packaged into large arrays and powered by readily available lasers with sub-nanosecond pulse widths. These two factors have enabled the development of GmAPD LiDAR systems that can collect very high density data with coverage rates well in excess of $1,000 \mathrm{~km} 2 / \mathrm{hr}$. Paired with advanced automation for high-volume processing and analytics, GmAPD sensors provide a revolutionary new capability for commercial environments.

This paper briefly describes GmAPD detector arrays, the basic design and operation of a Geiger-mode LiDAR developed by Harris Corp., and a high-volume production system with a focus on noise reduction and photogrammetric registration techniques.

\section{Why Use a GmAPD Sensor? - Efficiency and Maturity}

Unlike linear-mode systems, which proportionately convert photons to current signals, Geiger-mode detectors rely on an array of avalanche photodiodes (APDs) operating in an over-biased scenario such that they generate large, easily traceable currents that can be detected by digital timing circuitry when just a few photons are present. GmAPD detector arrays make excellent LiDAR receivers for the following reasons:

1. Low Timing Jitter - GmAPD arrays integrate simple CMOS digital timing circuits with the individual APDs. These respond to the leading edge of the avalanche current within 100 ps to record pulse time of flight, allowing for excellent range precision (ITZLER et al. 2010).

2. High Detection Sensitivity and Efficiency - Currently available GmAPD elements have photon detection efficiencies (PDEs) of up to $40 \%$. This means that a reflected pulse containing only $5-10$ photons is required for high probability detection - an order of magnitude less than is required by typical linear detectors. In addition, micro lenses are used to increase the effective detector fill factor to $\sim 75 \%$, ensuring every return photon incident on the array is efficiently coupled to a detector (ITZLER et al. 2010).

3. Compact Detectors - Because GmAPD detection elements require no analogue gain circuits, it is possible to produce compact LiDAR detector arrays. They enable increased sampling densities, which enable systems to sample the ground more rapidly, increasing coverage rates.

These three properties combined allow GmAPD sensors to fly faster, fly higher and scan wider swaths without sacrificing vertical accuracy. 


\section{ITI-1000 GmAPD LiDAR Sensor}

\section{Technical Design}

The ITI-1000 GmAPD LiDAR sensor is optimized for supporting USGS LiDAR Quality Level 1 (QL1, HEIDEMANN 2014) and higher data production over large areas, from pressurized, fixed-wing aircrafts flying at altitudes of between $4 \mathrm{~km}$ and $10 \mathrm{~km}$ AGL.

In those medium and high altitudes, a GmAPD LiDAR has a significant advantage over traditional linear-mode systems due to the single-photon sensitivity of the detectors. The traditional systems require at least an order of magnitude more photons for a detection, which in turn drives up the average power requirements and overall size, weight and power (SWaP) of the system, making a medium- or high-altitude linear-mode LiDAR impractical (CLIFTON et al. 2015).

Figure 1 shows the primary specifications and components of the system. "INS \& Control Electronics" denote the inertial navigation system (INS/GPS) including an inertial measurement unit (IMU). The patented coaxial transmit/receive Palmer scanner with $15^{\circ}$ scan half angle is capable of rotational speeds in excess of 2,000 RPM. The scan pattern is explained in Figure 2.

At the specified flight altitudes, it is critical that the pointing offset between transmit/receive scanners be adjustable to maintain optimal overlap between the transmitted beam and the received field of view (FOV). The ITI-1000 sensor uses a patent-pending technology to electronically steer the pointing offset on the fly. It also has automated gate control, and multiple-pulse-in-the-air tracking and compensation, critical features at the specified operational altitudes and pulse repetition frequencies (PRF).

\begin{tabular}{|c|c|}
\hline \multirow{2}{*}{\multicolumn{2}{|c|}{ ITI-1000 Technical Specifications }} \\
\hline Flight & \\
\hline Altitude (AGL) & 4-10 km (6 km nominal $)$ \\
\hline Flight Speed & $280-560 \mathrm{~km} / \mathrm{h}$ \\
\hline Swath Width & $2-5 \mathrm{~km}(3.2 \mathrm{~km}$ nominal $)$ \\
\hline \multicolumn{2}{|l|}{ Palmer Scanner } \\
\hline Scan Half Angle & $15^{\circ}$ \\
\hline Aperture Diameter & $27 \mathrm{~cm}$ \\
\hline \multicolumn{2}{|l|}{ Transmit Laser } \\
\hline Wavelength & $1064 \mathrm{~nm}$ (Class IV) \\
\hline Average Power & $20 \mathrm{~W}$ \\
\hline Pulse Width & $550 \mathrm{ps}$ \\
\hline Pulse Repetition Frequency & $50 \mathrm{kHz}$ \\
\hline $\mathrm{NOHD} / \mathrm{ENOHD}$ & $300 \mathrm{~m} / 2.2 \mathrm{~km}$ \\
\hline \multicolumn{2}{|l|}{ GmAPD Receiver } \\
\hline Array Size & $32 \times 128$ \\
\hline IFOV & $35 \mathrm{\mu rad}$ \\
\hline PDE & $30 \%$ \\
\hline Timing Resolution & $250-500 \mathrm{ps}$ \\
\hline \multicolumn{2}{|c|}{ Coverage Rate (with $50 \%$ overlap) } \\
\hline 4 points per $\mathrm{m}^{2}$ & $1100 \mathrm{~km}^{2} / \mathrm{h}$ \\
\hline 8 points per $\mathrm{m}^{2}$ & $1000 \mathrm{~km}^{2} / \mathrm{h}$ \\
\hline 20 points per $\mathrm{m}^{2}$ & $700 \mathrm{~km}^{2} / \mathrm{h}$ \\
\hline
\end{tabular}

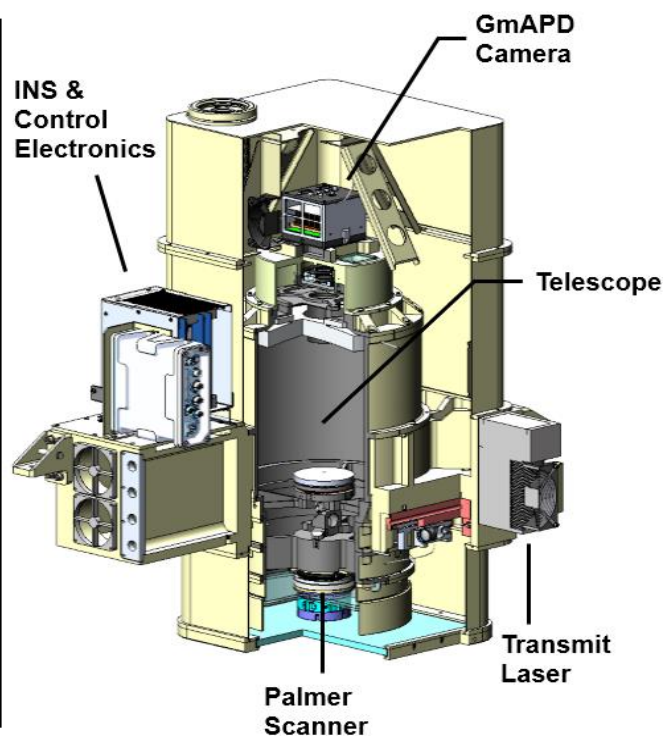

Figure 1: Specifications (left) and CAD diagram (right) for the ITI-1000 GmAPD sensor. 

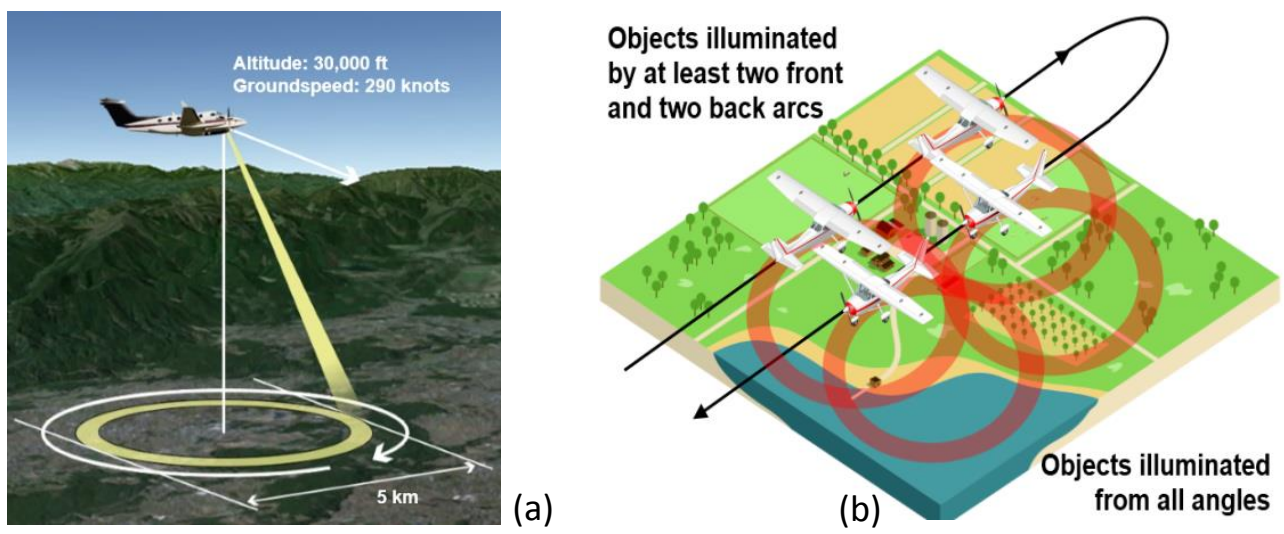

Figure 2: (a) The ITI-1000 GmAPD sensor uses a Palmer scanner to increase both aperture size and swath half angle. The back and front arcs of the scan are treated as individual swaths during production. (b) Each ground sample spot is sampled over four arcs - two backwards and two forwards - and from four different angles, to create a densely aggregated point cloud.

\section{Acquisition Operations}

Collecting data with a GmAPD sensor requires a slightly different approach than with current linear-mode sensors, since digitization is happening at the detector level. Consider the scenario where the sensor is imaging a flat surface at a fixed distance (Figure 3a). Typical linear-mode flash or spotlight LiDAR sensors use a single high-energy pulse to illuminate the scene. The light reflected back to the receiver is converted and amplified into an electric signal proportional to the incident light intensity (Figure 3b). Then, either single or multiple points per pulse are returned by finding the leading edge of peaks in the data, or the full waveform is digitally sampled and stored for later analysis. 


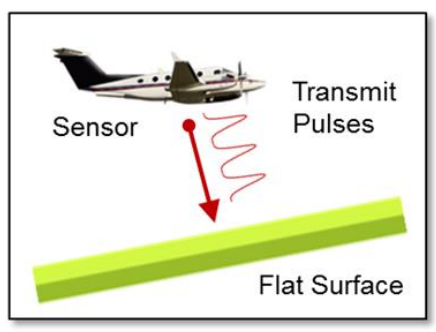

(a)

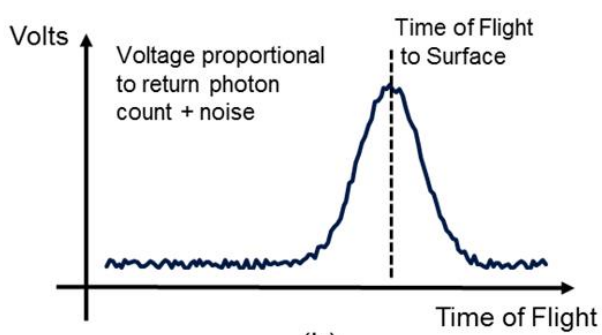

(b)

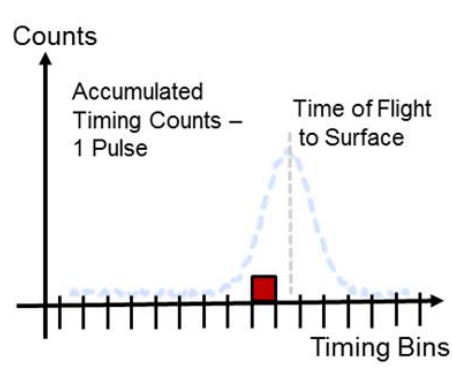

Counts

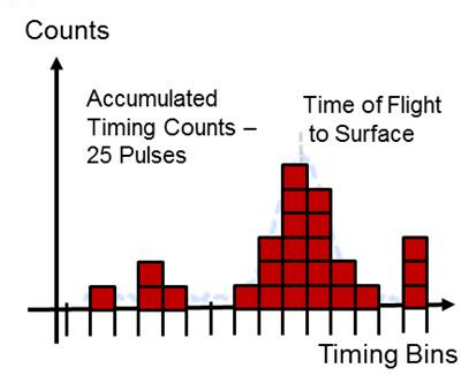

(c)

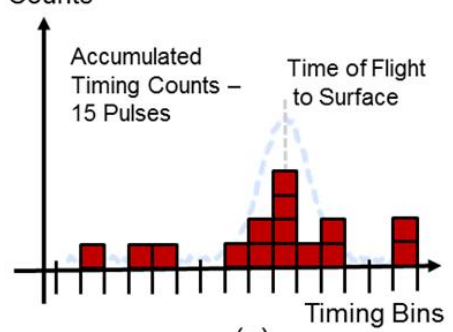

\section{Figure 3:}

(a) An airborne LiDAR sensor measuring the distance to a flat earth segment.

(b) A linear-mode sensor converts reflected pulse photons to a proportional electronic signal. Range is measured by finding the time difference between rising peaks of the transmit laser pulse and strong peaks in the electrical return signal.

(c) GmAPD sensors measure the target range by fitting an expected pulse shape to the accumulated timing counts from multiple laser firings and computing the same timing delta.

Due to the binary nature of the detectors, GmAPD LiDAR systems require the aggregation of multiple pulses to locate surfaces in the data. When a small number of photons (typically 5-10) impinge on a GmAPD detector, they cause an avalanche current which builds and, after about 100 ps, STOPS A CMOS TIMER (Itzler et al. 2010). This detector then remains blind until the array is reset and rearmed. As a result, as shown in Figure 3c, it is not possible to differentiate, from a single laser shot, between an actual surface and noise. To reliably detect surfaces, multiple pulses must be fired within the same ground instantaneous field of view (IFOV) and accumulated, their number being dependent on the expected detection probability of the surface. The sensor is programmed for each collection to ensure that the ground is sampled with the appropriate pulse count to meet resolution and density specifications. The relative reflectance of the surface can also be returned as the number of counts at the surface time range to produce a humanly interpretable grey scale image.

A set of acquisition procedures is applied when using the ITI-1000 sensor. First, each ground spot is sampled with the appropriate number of pulses for the desired resolution of the point cloud. The half-arcs of pulses form separate forward and backward swaths. Flight lines are laid out with at least $50 \%$ overlap after roll allowances, ensuring each ground sample area is illuminated from four different directions, reducing self-shadowing and increasing sample density (see Figure 2b). Data from all four of these arcs are aggregated, based on the desired point cloud resolution, to provide the best possible raw range data. 


\section{$4 \quad$ High-Volume LiDAR Processing}

\section{Automated-Processing Workflow for ITI-1000}

The workflow shown in Figure 4 was developed specifically for data acquired by the ITI-1000 GmAPD sensor and attempts to minimize the amount of work required by analysts in order to support large-scale collection and production without sacrificing data quality.

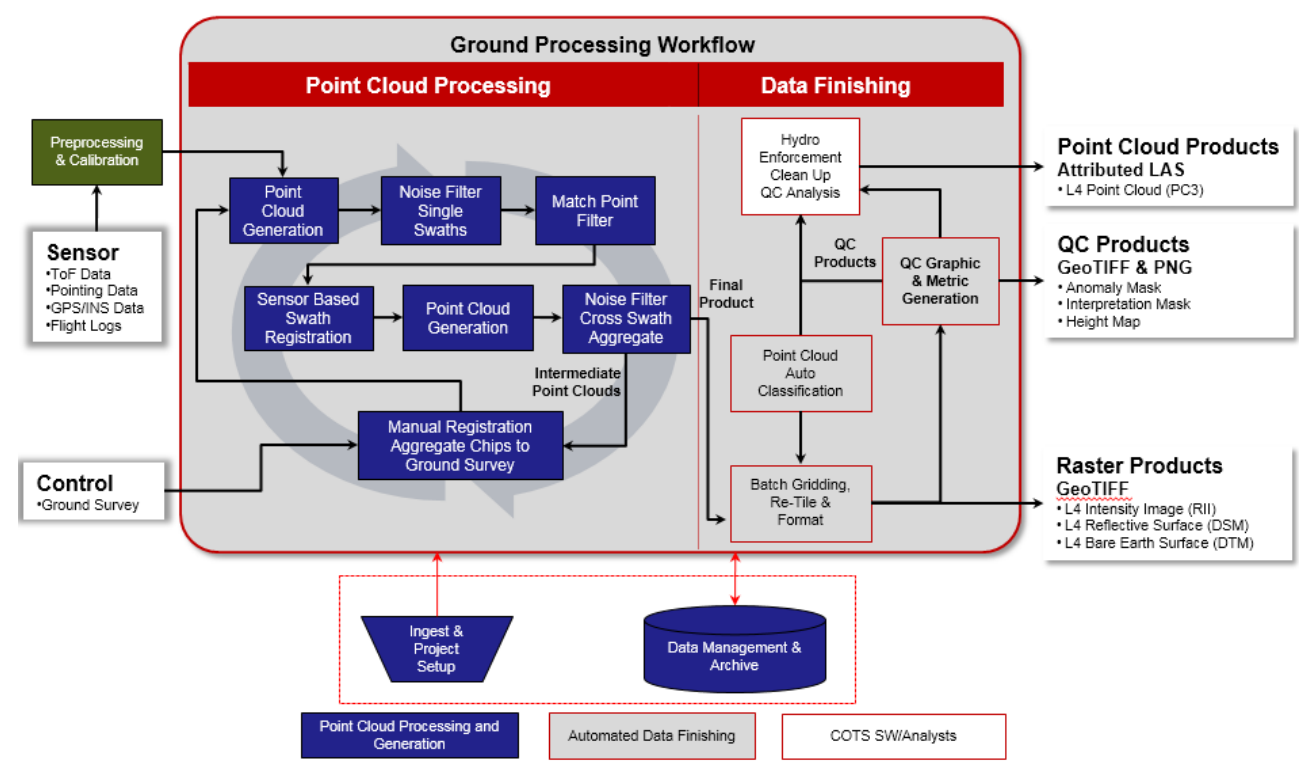

Figure 4: Automated workflow to support GmAPD processing and production. It uses noise filtering, photogrammetric registration, point labelling, and quality control analysis software developed by Harris Corp.

In the pre-processing phase, in order to improve accuracy, a combination of automatic and manual tools is used to perform sensor-calibration and non-causal adjustment of the trajectory of the GPS platform. The improved trajectory and sensor-calibration data are then sent, along with the raw-time sensor data, to the first stage of automated processing, where the range data are converted from angle-angle-time space of the sensor to 3D geodetic coordinates on a swath-by-swath basis. In the next phase, fast noise filtering is performed on each swath. Potential match points are then found between swaths and used to align the swaths via a sensor-model-based bundle adjustment that improves knowledge of the trajectory of the platform. The raw data is then re-projected from sensor space to 3D ground coordinates using the adjusted trajectory, and multi-swath aggregate point clouds are formed. These aggregate point clouds then undergo a sophisticated noise-filtering process to produce the final 3D point cloud products. 
The final 3D point clouds are then delivered to the data-finishing tool suite, which combines both automated and manual software tools to produce a wide range of products, including digital surface and terrain models, relative reflectance imagery, and other valueadded products, as required by the project.

\section{Noise Reduction for GmAPD Point Clouds}

Although analogue gain noise is not an issue, GmAPD detector arrays are highly susceptible to noise from other sources due to the highly sensitive nature of the detection technology (see Figure $5)$.

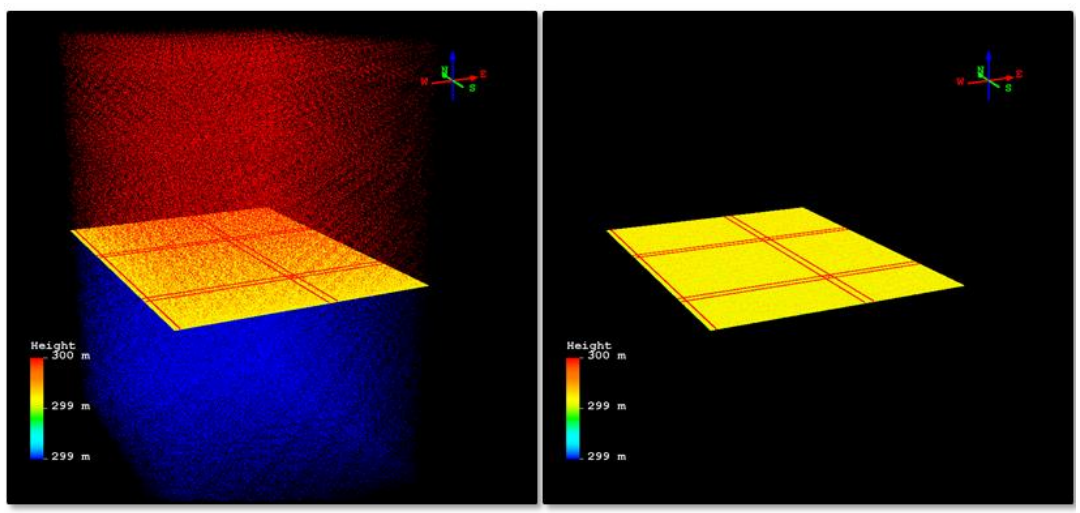

Figure 5: GmAPD point clouds contain a substantial amount of noise due to the high sensitivity of the detectors (left). The automated processing workflow for ITI-1000 contains algorithms for recovering the actual surface(s) from the noise (right).

The three primary sources of noise are the dark count rate of the device (i.e. the number of detections with no incident light), cross-talk between detectors (stray photons generated due to the avalanche process), and the level of solar background signal present in the scene, a noise source common to time-of-flight LiDAR systems (Smith \& Clifton 2012). The total noise rate is dominated by the background rate during the day, which is typically two orders of magnitude greater than the dark count rate or level of cross-talk. Solar background noise is reduced by using a narrow band interference filter centred on the receiver's transmit laser wavelength, and by reducing the range gate duration to the minimum necessary to image the scene.

As a result, recovering clean 3D point clouds from GmAPD requires not only techniques for creating aggregate images (as discussed in section 4.1), but also sophisticated filtering techniques for reducing/removing noise from the signals.

\section{Accuracy Improvements}

As mentioned above, Geiger-mode sensors provide significant collection efficiency advantages compared to linear-mode systems. They do, however, require more accurate 
platform trajectory information, due to the fact that they produce point clouds via multipulse aggregation.

While the ITI-1000 sensor employs the latest in GPS technology, production of high-quality, accurate data from this or any other GmAPD system requires true 3D registration of data from individual swaths. Harris Corp. technology allows true sensor-model bundle adjustment using photogrammetric registration techniques driven by aligning automatically-matched tie points across swaths (see Figure 6).

\section{Multi-Swath Alignment via Sensor-Based} 3D Photogrammetric Bundle Adjustment

\section{Enables Rigorous Accuracy Statements per Point}
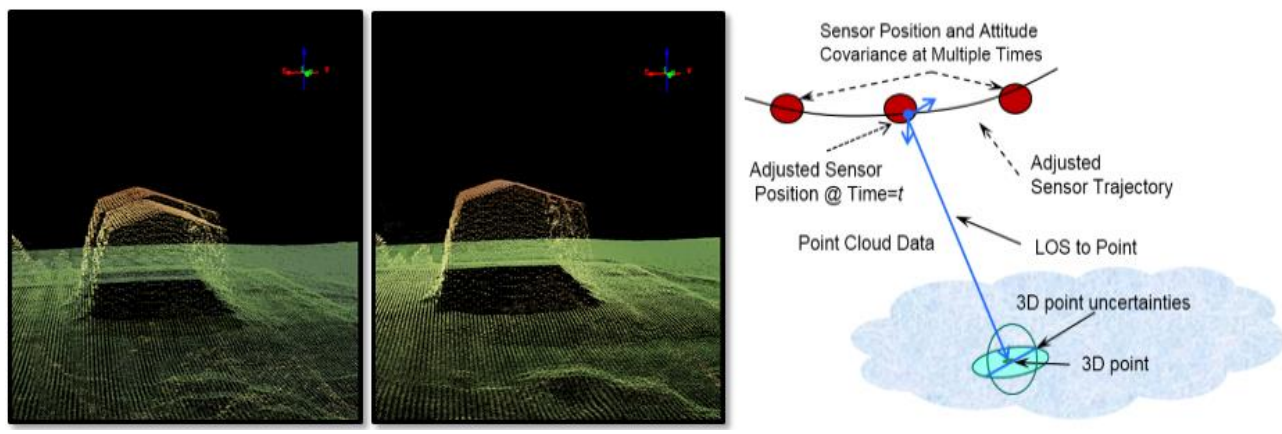

Figure 6: (Left) Unregistered aggregate GmAPD point cloud data of a building taken from two adjacent flight lines. The building and ground are misaligned, primarily due to inaccuracies in the INS trajectory. (Middle) Aggregated point cloud after full 3D bundle adjustment. The building and ground are now aligned due to improvement in trajectory knowledge obtained via sensor model-based registration. (Right) Sensor-based bundle adjustment allows the delivery of per-point accuracy statements with its LiDAR data products.

This technique also allows the integration of ground control points into the best and final production process for a given collection area by manually locating ground survey points in the data and using them as fixed points in a final bundle adjustment.

\section{Conclusion}

Over the past 15 years, several LiDAR imaging sensors based on Geiger-mode avalanche photodiode (GmAPD) arrays have been successfully flown, providing the technical foundation for a commercial Geiger-mode LiDAR mapping system. The extreme sensitivity of GmAPD detectors allows operation of LiDAR sensors at unprecedented altitudes and area collection rates in excess of $1,000 \mathrm{~km} 2 / \mathrm{hr}$ (Clifton et al. 2015).

The Geiger-mode LiDAR sensor developed by Harris Corp. is the first commercial airborne LiDAR system that takes advantage of the GmAPD capabilities, applying this technology to non-military uses such as mapping, monitoring critical infrastructure, and disaster relief. 
Geiger-mode data sets are much larger than those collected by linear-mode systems. As a result, a processing engine has been developed that uses advanced blade server technology and an automated workflow to handle these enormous data sets quickly. In the present state, the sensor is currently provided as a service, offering its own hardware, airplanes, and processing methods to deliver the data.

\section{References}

Cary, T. (2009). LiDAR Market: Status and Growth Trends. International LiD AR Mapping Forum. Denver, CO.

Clifton, W. E., Steele, B., Nelson, G., Truscott, A., Itzler, M., Entwistle, M. (2015). Medium Altitude Airborne Geiger-mode Mapping Lidar System. Proc. SPIE 2015 9465, 8 p. doi: $10.1117 / 12.2193827$

Heidemann, H. K. (2014). Lidar base specification. USGS Techniques and Methods, 11-B4, 67 p. doi: 10.3133/tm11B4

Itzler, M., Entwistle, M., Owens, M., Patel, K., Jiang, X., Slomkowski, K., Ferrar, J. (2010). Geigermode avalanche photodiode focal plane arrays for three-dimensional imaging LADAR. Proc. SPIE 20107808,14 p. doi: 10.1117/12.861600

Smith, P. W., Clifton, W. E. (2012). An Overview of ALS Technology: Geiger-Mode LIDAR. In Renslow, M. [Ed.], Manual of Airborne Topographic LIDAR (pp. 91-96). Behtesda, MD, ASPRS. 